\title{
Avaliação da Qualidade de Vida em Pacientes Submetidos à Ressecção Colorretal por Via Laparoscópica ou Aberta em Período Pós-Operatório Inicial
}

\author{
Evaluation of Quality of Life in Patients Underwent Laparoscopic or Open \\ Colorectal Resection in the Early Postoperative Period
}

\begin{abstract}
TEON AUGUSTONORONHA DE OLIVEIRA ${ }^{1}$; FÁBIOLOPES DEQUEIROZ ${ }^{1-2}$; ANTÔNIOLACERDA-FILHO $^{1-3-4}$; ELIANE SANDER MANSUR ${ }^{1}$; MARIA ZULEIME CARMONA ${ }^{1}$; MARCELO SANTOS RESENDE ${ }^{6}$; PAULOCÉSAR DECARVALHOLAMOUNIER ${ }^{1}$; RODRIGO DE ALMEIDA PAIVA ${ }^{1}$; VALDIVINOALVES FILHO ${ }^{1}$
\end{abstract}

\begin{abstract}
${ }^{1}$ Médico Residente da Clínica de Coloproctologia do Hospital Felício Rocho. Belo Horizonte/MG; ${ }^{2}$ Mestre em Genética pelo Instituto de Ciências Biológicas da Universidade Federal de Minas Gerais. Belo Horizonte/MG; ${ }^{3}$ Professor Assistente do Departamento de Cirurgia da Faculdade de Medicina da UFMG. ${ }^{4}$ Membro Titular do Grupo de Coloproctologia e Intestino Delgado do Instituto Alfa de Gastroenterologia do Hospital das Clínicas da UFMG. Belo Horizonte/MG; ${ }^{5}$ Médico Assistente da Clínica de Coloproctologia do Hospital Governador Israel Pinheiro; ${ }^{6}$ Médico Residente do Hospital Governador Israel Pinheiro.
\end{abstract}

OLIVEIRA TAN; QUEIROZ FL; LACERDA-FILHO A; MANSUR ES; CARMONA MZ; RESENDE MSLAMOUNIER PCC; PAIVA RA; ALVES FILHO V. Avaliação da Qualidade de Vida em Pacientes Submetidos à Ressecção Colorretal por Via Laparoscópica ou Aberta em Período Pós-Operatório Inicial. Rev bras Coloproct, 2010;30(1): 037-044.

RESUMO: Introdução: A publicação de estudos controlados e metanálises que evidenciaram a segurança, a efetividade e a equivalência oncológica da abordagem laparoscópica com relação aos procedimentos abertos, fez com que a cirurgia colorretal minimamente invasiva fosse adotada por um número crescente de serviços em todo o mundo. O objetivo desse estudo foi avaliar se há diferenças na qualidade de vida dos pacientes no período pós-operatório inicial de cirurgias colorretais laparoscópicas e convencionais. Pacientes e Método: Trata-se de um estudo prospectivo observacional sobre qualidade de vida, envolvendo pacientes submetidos à cirurgia colorretal laparoscópica e convencional. No período de maio a novembro de 2008, 42 pacientes submetidos à cirurgia colorretal foram acompanhados desde a cirurgia até o $60^{\circ}$ DPO. Os questionários foram aplicados no $3^{\circ}, 7^{\circ}$ e $30^{\circ}$ dias de pós-operatório e as respostas obtidas constituíram o banco de dados a ser analisado. A análise estatística consistiu em análise descritiva do escore do estado global de saúde, dos escores funcionais e de sintomas do questionário EORTC/QLQ 30, tendo sido utilizado os testes de Shapiro-Wilk, Mann-Whitney e t de Student. $O$ nível de significância (a) considerado foi de 0,05 . Resultados: Houve predominância de pacientes do sexo feminino $\left(57,1 \%\right.$ ), com uma média de idade de 61,5 anos. Foi detectada diferença significativa do escore "estado global de saúde" no $30^{\circ}$ dia de pósoperatório, com valores de $\mathbf{7 5 , 0}$ e 58,3 para as cirurgias laparoscópicas e abertas, respectivamente $(\mathbf{p}=\mathbf{0 , 0 0 5})$. Com relação à função física, assim como na análise das demais funções (desempenho, emocional, cognitiva e social), sintomas (fadiga, náusea, dor, dispnéia, insônia, apetite, constipação) e dificuldades financeiras, não foram observadas diferenças significativas. Conclusão: Os resultados demonstraram que os pacientes submetidos à cirurgia colorretal por via laparoscópica apresentaram melhor qualidade de vida ao final do primeiro mês de pós-operatório, quando comparados com os pacientes submetidos à cirurgia aberta.

Descritores: Qualidade de vida; câncer colorretal; cirurgia laparoscópica.

\section{INTRODUÇÃO}

A ressecção laparoscópica colorretal é hoje uma alternativa à cirurgia convencional. A publicação de estudos controlados e metanálises que evidenciaram a segurança, a efetividade e a equivalência oncológica da abordagem laparoscópica quando comparada aos procedimentos por via aberta fez com que

Trabalho realizado na Clínica Coloproctológica do Hospital Felício Rocho - Belo Horizonte/MG.

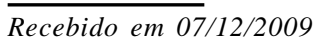

Aceito para publicação em 28/12/2009 
a cirurgia colorretal minimamente invasiva fosse adotada por um numero crescente de serviços em todo o mundo.

Vários benefícios da abordagem laparoscópica no pós-operatório imediato têm sido demonstrados ${ }^{1}$, embora também tenham sido relatadas poucas diferenças e até mesmo desvantagens desta via, como aumento do tempo cirúrgico, maior custo do procedimento e a necessidade de uma longa curva de aprendizado $^{2,3}$. Por outro lado, metanálises recentes têm demonstrado que a cirurgia colorretal laparoscópica está associada a um menor período de hospitalização, menor dor pós-operatória, menor duração do íleo paralítico, retorno precoce ao trabalho e a um melhor resultado estético ${ }^{4}$.

O exato efeito destas duas abordagens na qualidade de vida dos pacientes no período pós-operatório ainda não está bem definido na literatura médica. A maioria dos artigos de revisão não estabelece diferença na qualidade de vida entre os pacientes submetidos à cirurgia por via laparoscópica e por via aberta. Sabese que parece haver uma tendência a melhora da qualidade de vida nos pacientes submetidos à cirurgia minimamente invasiva ${ }^{5,6}$.

O objetivo desse estudo foi avaliar se há diferenças na qualidade de vida dos pacientes submetidos à cirurgia colorretal por via laparoscópica e por via aberta no período pós-operatório inicial.

\section{PACIENTES E MÉTODOS}

Trata-se de um estudo prospectivo observacional, envolvendo 42 pacientes submetidos à cirurgia colorretal por via laparoscópica ou por via aberta no período de maio a novembro de 2008 e que foram acompanhados desde a cirurgia até o $60^{\circ} \mathrm{DPO}$.

Os pacientes foram distribuídos em dois grupos. O grupo I foi submetido à cirurgia laparoscópica e o Grupo II, a cirurgia convencional, isto é, por via aberta. A distribuição dos pacientes pelos grupos I e II se deu exclusivamente a partir da preferência do cirurgião pela via de acesso. Foram incluídos apenas pacientes maiores de 18 anos de idade. Pacientes em que houve conversão para cirurgia aberta durante o procedimento laparoscópico foram excluídos do estudo.

Dos 42 pacientes, 15 foram submetidos à cirurgia laparoscópica e 27 à cirurgia convencional. Esses pacientes responderam a questionário de avalia- ção da qualidade de vida validado na literatura (questionário de qualidade de vida da organização européia para pesquisa e tratamento do câncer (EORTC) - QLQ$\mathrm{C} 30)^{7}$.

Os questionários foram aplicados no $3^{\circ}, 7^{\circ} \mathrm{e}$ $30^{\circ}$ dias de pós-operatório e os dados foram coletados através das respostas obtidas e organizados em banco de dados, segundo as variáveis de interesse em cada fase da análise estatística. Tais dados forneceram resultados relativos à evolução e ao benefício em relação à qualidade de vida dos pacientes submetidos à cirurgia laparoscópica e convencional.

O questionário utilizado avalia a qualidade de vida em pacientes oncológicos. Este questionário apresenta 30 questões que combinadas avaliam sintomas, atividades e a qualidade de vida. Os pacientes responderam aos questionários através de entrevistas realizadas pessoalmente ou por contato telefônico, sempre pelo mesmo entrevistador.

A análise estatística consistiu em análise descritiva do escore do estado global de saúde, dos escores funcionais e de sintomas do questionário EORTC/ QLQ 30. Na verificação da normalidade dos escores, foi utilizado o teste de Shapiro-Wilk. Na comparação de medianas foi utilizado o teste não paramétrico de Mann-Whitney e na comparação de médias, o teste $t$ de Student para amostras independentes. Para os escores que não seguiram distribuição normal, a mediana foi utilizada como a melhor medida de tendência central, sendo que os testes utilizados nas comparações envolvendo as mesmas foram os não paramétricos, no caso o teste de Mann-Whitney.

O nível de significância (a) considerado foi de 0,05 . O pacote estatístico utilizado foi o SPSS 15.0 for Windows.

Esse estudo foi avaliado e aprovado pelo Comitê de Ética em Pesquisa do Hospital Felício Rochoprotocolo $\mathrm{n}^{\circ}$ 242/08.

\section{RESULTADOS}

Dos 42 pacientes, $24(57,1 \%)$ eram mulheres. A média de idade foi de 61,5 anos (variação de 40 a 81). Sete pacientes não responderam a algum dos questionários. Destes, dois não foram avaliados no $3^{\circ}$ dia de pós-operatório por estarem internados no centro de tratamento intensivo e os outros cinco pacientes desistiram de participar do estudo após responderem o questionário do $3^{\circ}$ dia de pós-operatório, não respondendo 
aos questionários no $7^{\circ}$ e no $30^{\circ}$ dias de pós-operatório.

As tabelas 1, 2 e 3 apresentam o teste de Shapiro-Wilk para verificação de normalidade dos escores no $3^{\circ}, 7^{\circ}$ e $30^{\circ}$ dias após a cirurgia. A média do escore "estado global de saúde" no $3^{\circ}$ dia para a técnica convencional foi igual a 54 e para a técnica laparoscópica foi igual a 58,3 ( $\mathrm{p}=\mathrm{NS}$ ). Para os escores "Estado Global de Saúde" no sétimo dia também não foi detectada diferença estatisticamente significativa das medianas $(\mathrm{p}=0,205)$. Foi detectada dife- rença significativa da mediana do escore "Estado Global de Saúde" no $30^{\circ}$ dia após a cirurgia em função da técnica cirúrgica $(p=0,005)$, uma vez que para os pacientes que realizaram cirurgia convencional a mediana foi 58,3 e para os que realizaram cirurgia laparoscópica esses valor foi de 75,0. Portanto, com um mês de cirurgia, os pacientes submetidos ao procedimento por via laparoscópica apresentaram melhor qualidade de vida representada por escores mais elevados do "estado global de saúde" (Tabela 4 e Figura 1).

Tabela 1 - Teste de Shapiro-Wilk para verificação de normalidade dos escores obtidos no $3^{o}$ dia após a cirurgia.

\begin{tabular}{llcl}
\hline Escore $\mathbf{3}^{\circ}$ dia & Técnica Cirúrgica & Estatística Shapiro-Wilk & Valor p \\
\hline Estado Global de Saúde & Convencional & 0,978 & 0,840 \\
Função Física & Laparoscópica & 0,945 & 0,445 \\
& Convencional & 0,901 & $0,020^{*}$ \\
Desempenho de papéis & Laparoscópica & 0,918 & 0,177 \\
& Convencional & 0,817 & $0,000^{*}$ \\
Função Emocional & Laparoscópica & 0,848 & $0,016^{*}$ \\
Função Cognitiva & Convencional & 0,865 & $0,003^{*}$ \\
Função Social & Laparoscópica & 0,887 & 0,061 \\
& Convencional & 0,816 & $0,000^{*}$ \\
Fadiga & Laparoscópica & 0,886 & 0,059 \\
\multirow{2}{*}{ Náuse a e vômito } & Convencional & 0,908 & $0,027^{*}$ \\
& Laparoscópica & 0,854 & $0,020^{*}$ \\
Dor & Convencional & 0,945 & 0,195 \\
Dispnéia & Laparoscópica & 0,949 & 0,515 \\
& Convencional & 0,790 & $0,000^{*}$ \\
Insônia & Laparoscópica & 0,731 & $0,001^{*}$ \\
& Convencional & 0,897 & $0,015^{*}$ \\
Falta de Apetite & Laparoscópica & 0,846 & $0,015^{*}$ \\
Constipação & Convencional & 0,617 & $0,000^{*}$ \\
Diarréia & Laparoscópica & 0,284 & $0,000^{*}$ \\
Dificuldades Financeiras & Convencional & 0,858 & $0,003^{*}$ \\
& Laparoscópica & 0,789 & $0,003^{*}$ \\
& Convencional & 0,805 & $0,000^{*}$ \\
& Laparoscópica & 0,882 & 0,052 \\
& Convencional & 0,710 & $0,000^{*}$ \\
& Laparoscópica & 0,720 & $0,000^{*}$ \\
& Convencional & 0,357 & $0,000^{*}$ \\
& Laparoscópica & 0,771 & $0,002^{*}$ \\
& Convencional & 0,763 & $0,000^{*}$ \\
\hline
\end{tabular}

*Não segue distribuição normal. 
Tabela 2 - Teste de Shapiro-Wilk para verificação de normalidade dos escores obtidos no $7^{o}$ dia após a cirurgia.

\begin{tabular}{llcl}
\hline Escore $-\mathbf{7}^{\circ}$ dia & Técnica Cirúrgica & Estatística Shapiro-Wilk & Valor p \\
\hline Estado Global de Saúde & Convencional & 0,967 & 0,615 \\
Função Física & Laparoscópica & 0,843 & $0,018^{*}$ \\
Desempenho de papéis & Convencional & 0,888 & $0,015^{*}$ \\
& Laparoscópica & 0,922 & 0,236 \\
Função Emocional & Convencional & 0,820 & $0,001^{*}$ \\
Função Cognitiva & Laparoscópica & 0,916 & 0,190 \\
& Convencional & 0,882 & $0,011^{*}$ \\
Função Social & Laparoscópica & 0,855 & $0,026^{*}$ \\
& Convencional & 0,858 & $0,004^{*}$ \\
Fadiga & Laparoscópica & 0,749 & $0,001^{*}$ \\
\multirow{2}{*}{ Náuse a e vômito } & Convencional & 0,862 & $0,004^{*}$ \\
& Laparoscópica & 0,916 & 0,191 \\
Dor & Convencional & 0,967 & 0,611 \\
Dispnéia & Laparoscópica & 0,931 & 0,313 \\
Insônia & Convencional & 0,568 & $0,000^{*}$ \\
& Laparoscópica & 0,616 & $0,000^{*}$ \\
Falta de Apetite & Convencional & 0,922 & 0,075 \\
Constipação & Laparoscópica & 0,905 & 0,134 \\
Diarréia & Convencional & 0,621 & $0,000^{*}$ \\
Dificuldades Financeiras & Laparoscópica & 0,428 & $0,000^{*}$ \\
& Convencional & 0,853 & $0,003^{*}$ \\
& Laparoscópica & 0,758 & $0,002^{*}$ \\
& Convencional & 0,860 & $0,004^{*}$ \\
& Laparoscópica & 0,763 & $0,002^{*}$ \\
& Convencional & 0,621 & $0,000^{*}$ \\
& Laparoscópica & 0,751 & $0,001^{*}$ \\
& Convencional & 0,625 & $0,000^{*}$ \\
& Laparoscópica & 0,696 & $0,000^{*}$ \\
& Convencional & 0,821 & $0,001^{*}$ \\
& Laparoscópica & 0,714 & $0,001^{*}$ \\
\hline
\end{tabular}

*Não segue distribuição normal.

Com relação à função física, não foram detectadas diferenças significativas em função da técnica em nenhum dos dias. Nota-se que apesar da diferença não ter sido estatisticamente significativa, escores maiores no $7^{\circ}$ e $30^{\circ}$ dias de pós-operatório para a técnica laparoscópica, sugerem uma melhor função física para os pacientes deste grupo (tabela 5).

$\mathrm{Na}$ análise das demais funções (desempenho, emocional, cognitiva e social), sintomas (fadiga, náusea, dor, dispnéia, insônia, apetite, constipação), e dificuldades financeiras, não houve diferença estatisticamente significativa entre os grupos.

\section{DISCUSSÃO}

Este trabalho prospectivo observacional comparou cirurgias colorretais laparoscópicas com cirurgias convencionais através da análise de variáveis funcionais e variáveis relacionadas à sintomatologia e à qualidade de vida. Foi demonstrado benefício da técnica laparoscópica sobre a técnica convencional em relação ao "estado global de saúde" dos pacientes no $30^{\circ}$ dia de pós-operatório.

Estudo realizado por Schwenk e Muller ${ }^{8}$ também demonstrou diferença significativa na qualidade 
Tabela 3 - Teste de Shapiro-Wilk para verificação de normalidade dos escores obtidos no 30º dia após a cirurgia.

\begin{tabular}{llcl}
\hline Escore - 30 ${ }^{\circ}$ dia & Técnica Cirúrgica & Estatística Shapiro-Wilk & Valor p \\
\hline Estado Global de Saúde & Convencional & 0,907 & $0,041^{*}$ \\
Função Física & Laparoscópica & 0,954 & 0,625 \\
& Convencional & 0,946 & 0,258 \\
Desempenho de papéis & Laparoscópica & 0,814 & $0,008^{*}$ \\
& Convencional & 0,904 & $0,036^{*}$ \\
Função Emocional & Laparoscópica & 0,787 & $0,003^{*}$ \\
Função Cognitiva & Convencional & 0,880 & $0,012^{*}$ \\
& Laparoscópica & 0,801 & $0,005^{*}$ \\
Função Social & Convencional & 0,710 & $0,000^{*}$ \\
& Laparoscópica & 0,719 & $0,001^{*}$ \\
Fadiga & Convencional & 0,883 & $0,014^{*}$ \\
Náuse a e vômito & Laparoscópica & 0,861 & $0,032^{*}$ \\
Dor & Convencional & 0,860 & $0,005^{*}$ \\
Dispnéia & Laparoscópica & 0,809 & $0,006^{*}$ \\
Insônia & Convencional & 0,417 & $0,000^{*}$ \\
& Laparoscópica & 0,428 & $0,000^{*}$ \\
Falta de Apetite & Convencional & 0,751 & $0,000^{*}$ \\
Constipação & Laparoscópica & 0,668 & $0,000^{*}$ \\
Diarréia & Convencional & 0,522 & $0,000^{*}$ \\
Dificuldades Financeiras & Laparoscópica & - & - \\
& Convencional & 0,709 & $0,000^{*}$ \\
& Laparoscópica & 0,627 & $0,000^{*}$ \\
& Convencional & 0,596 & $0,000^{*}$ \\
& Laparoscópica & 0,627 & $0,000^{*}$ \\
& Convencional & 0,596 & $0,000^{*}$ \\
& Laparoscópica & 0,616 & $0,000^{*}$ \\
& Convencional & 0,647 & $0,000^{*}$ \\
& Laparoscópica & 0,503 & $0,000^{*}$ \\
& Convencional & 0,751 & $0,014^{*}$ \\
\hline
\end{tabular}

*Não segue distribuição normal.

**O valor do escore dispnéia para os pacientes da cirurgia laparoscópica foi constante.

de vida entre o grupo laparoscópico e o convencional. Por outro lado, tal benefício não vai de encontro aos resultados de estudos, como o CLASSIC e o $\operatorname{COST}^{9,10}$, que não evidenciam diferenças em termos de qualidade de vida entre pacientes submetidos a colectomias por via laparoscópica e aberta. Revisão recente de Dowson e cols ${ }^{5}$ também sugere que não haveria vantagens significativas na comparação da abordagem laparoscópica com a técnica aberta em termos de qualidade de vida.
Há vários estudos que demonstraram menor dor pós-operatória com a utilização da técnica laparoscópica. Recentemente, Lourenço e cols ${ }^{6}$ publicaram metanálise com 4500 pacientes que confirmou tal evidência. Contrariando estes resultados, o presente estudo não evidenciou diferença significativa entre a abordagem laparoscópica e a técnica aberta em relação à dor, embora tenham sido observados valores menores para este escore no grupo laparoscópico, como mostrado na tabela 6 e na figura 2 . Talvez com uma 
Tabela 4 - Distribuição do escore "Estado Global de Saúde" no $3^{\circ}, 7^{\circ}$ e $30^{\circ}$ dia após a cirurgia em função da técnica cirúrgica.

\begin{tabular}{|c|c|c|c|c|c|c|c|c|c|c|c|}
\hline Variável & $\begin{array}{l}\text { Técnica } \\
\text { Cirúrgica }\end{array}$ & $\mathbf{N}$ & Média & $\begin{array}{l}\text { Desvio } \\
\text { Padrão }\end{array}$ & Mínimo & Máximo & $\begin{array}{c}1^{\circ} \\
\text { quartil }\end{array}$ & Mediana & $\begin{array}{c}3^{\mathbf{0}} \\
\text { quartil }\end{array}$ & Estatística & Valorp \\
\hline Estado Global & Convencional & 25 & 54,0 & 24,3 & 0,0 & 100,0 & 33,3 & 50,0 & 66,7 & $-0,588$ & 0,560 \\
\hline de Saúde $3^{\circ} \mathrm{DPO}$ & Laparoscópica & 15 & 58,3 & 19,2 & 33,3 & 100,0 & 41,7 & 58,3 & 66,7 & & \\
\hline Estado Global de & Convencional & 23 & 54,0 & 25,1 & 8,3 & 100,0 & 33,3 & 58,3 & 66,7 & 121,0 & 0,205 \\
\hline Saúde $7^{\circ} \mathrm{DPO} * *$ & Laparoscópica & 14 & 63,7 & 22,3 & 8,3 & 83,3 & 50,0 & 66,7 & 83,3 & & \\
\hline Estado Global de & Convencional & 22 & 58,7 & 15,5 & 16,7 & 83,3 & 50,0 & 58,3 & 66,7 & 68,5 & $0,005 *$ \\
\hline Saúde $30^{\circ} \mathrm{DPO} * *$ & Laparoscópica & 14 & 75,6 & 15,5 & 50,0 & 100,0 & 64,6 & 75,0 & 85,4 & & \\
\hline
\end{tabular}

* Estatisticamente significativo.

** Teste de Mann-Whitney

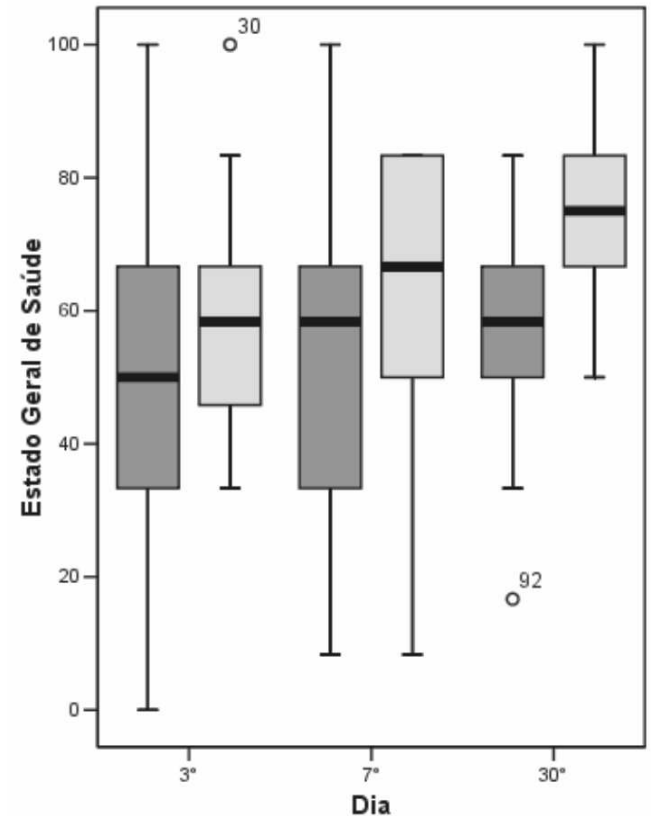

Figura 1 - Box-plot do Estado Geral de Saúde em função da técnica e do dia.

Procedimento ㅁ Convencional 口 Laparoscópico
Tabela 5 - Distribuição do escore "Função Física" no 3, 7o e 30o dia após a cirurgia em função da técnica cirúrgica.

\begin{tabular}{|c|c|c|c|c|c|c|c|c|c|c|c|}
\hline Variável & $\begin{array}{l}\text { Técnica } \\
\text { Cirúrgica }\end{array}$ & $\mathbf{N}$ & Média & $\begin{array}{l}\text { Desvio } \\
\text { Padrão }\end{array}$ & Mínimo & Máximo & $\begin{array}{c}1^{\circ} \\
\text { quartil }\end{array}$ & Mediana & $\begin{array}{c}3^{\circ} \\
\text { quartil }\end{array}$ & $\begin{array}{l}\text { Ude Mann- } \\
\text { Whitney }\end{array}$ & Valorp \\
\hline Função Física & Convencional & 25 & 53,6 & 34,5 & 0,0 & 100,0 & 26,7 & 53,3 & 93,3 & 171,0 & 0,643 \\
\hline $3^{\circ} \mathrm{DPO} * *$ & Laparoscópica & 15 & 47,6 & 30,1 & 0,0 & 93,3 & 13,3 & 40,0 & 73,3 & & \\
\hline Função Física & Convencional & 23 & 54,5 & 35,4 & 0,0 & 100,0 & 26,7 & 46,7 & 100,0 & 144,5 & 0,603 \\
\hline $7^{\circ} \mathrm{DPO} * *$ & Laparoscópica & 14 & 61,4 & 26,8 & 13,3 & 100,0 & 40,0 & 63,3 & 86,7 & & \\
\hline Função Física & Convencional & 22 & 71,2 & 22,0 & 20,0 & 100,0 & 53,3 & 73,3 & 88,3 & 107,5 & 0,128 \\
\hline $30^{\circ} \mathrm{DPO} * *$ & Laparoscópica & 14 & 81,0 & 22,5 & 20,0 & 100,0 & 71,7 & 86,7 & 100,0 & & \\
\hline
\end{tabular}

casuística maior, tal diferença poderia ter sido estatisticamente significativa, indo de encontro aos resultados publicados na literatura.

Da mesma maneira, em relação à variável função física, notou-se que os valores do escore da técnica laparoscópica eram maiores em comparação com o escore da abordagem convencional, sugerindo uma melhor função com o uso desta via de acesso. Novamente, considera-se que essa tendência poderia atingir significância estatística, caso a casuística fosse maior.

Considerando os resultados das outras treze variáveis do estudo, não se evidenciou diferenças estatisticamente significativas entre os grupos. Também não houve diferença entre as características dos pacientes estudados. Tanto o grupo submetido à cirurgia laparoscópica, quanto o grupo submetido à abordagem convencional foram semelhantes em relação à idade, sexo, sítio da neoplasia e ao tipo de ressecção. Isto demonstra que a cirurgia colorretal por via

** Teste de Mann-Whitney. 
Tabela 6 - Distribuição do escore "Dor" no $3^{\circ}, 7^{\circ}$ e $30^{\circ}$ dia após a cirurgia em função da técnica cirúrgica.

\begin{tabular}{|c|c|c|c|c|c|c|c|c|c|c|c|}
\hline Variável & $\begin{array}{l}\text { Técnica } \\
\text { Cirúrgica }\end{array}$ & $\mathbf{N}$ & Média & $\begin{array}{l}\text { Desvio } \\
\text { Padrão }\end{array}$ & Mínimo & Máximo & $\begin{array}{c}1^{\circ} \\
\text { quartil }\end{array}$ & Mediana & $\begin{array}{c}3^{\circ} \\
\text { quartil }\end{array}$ & Estatística & Valorp \\
\hline \multirow[t]{2}{*}{ Dor $3^{\circ} \mathrm{DPO} * *$} & Convencional & 25 & 48,0 & 35,1 & 0,0 & 100,0 & 16,7 & 50,0 & 75,0 & 136,0 & 0,145 \\
\hline & Laparoscópica & 15 & 32,2 & 32,4 & 0,0 & 83,3 & 0,0 & 33,3 & 66,7 & & \\
\hline \multirow[t]{2}{*}{ Dor $7^{\circ} \mathrm{DPO}$} & Convencional & 23 & 49,3 & 32,4 & 0,0 & 100,0 & 16,7 & 50,0 & 66,7 & 1,345 & 0,187 \\
\hline & Laparoscópica & 14 & 34,5 & 32,3 & 0,0 & 100,0 & 0,0 & 33,3 & 54,2 & & \\
\hline \multirow[t]{2}{*}{ Dor $30^{\circ}$ DPO $* *$} & Convencional & 22 & 20,5 & 27,7 & 0,0 & 100,0 & 0,0 & 16,7 & 33,3 & 121,0 & 0,242 \\
\hline & Laparoscópica & 14 & 9,5 & 14,2 & 0,0 & 33,3 & 0,0 & 0,0 & 20,8 & & \\
\hline
\end{tabular}

**Teste de Mann-Whitney.

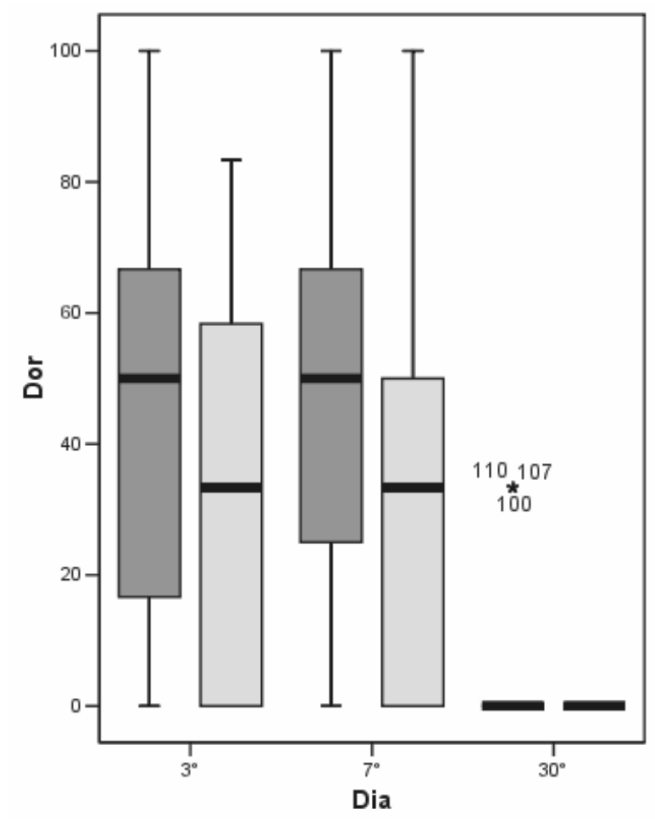

Figura 2 - Box-plot do Escore de dor entre os grupos laparoscópico e convencional no 3o e 7o dias de pós-operatório.

Procedimento C Convencional $\square$ Laparoscópico

\footnotetext{
ABSTRACT: Introduction: Several studies, including meta-analysis, have demonstrated the safety, effectiveness and oncologic equivalence of laparoscopic resections when compared to open procedures leading minimally invasive colorectal surgery to be adopted in crescent number of services around the world. This study aims to evaluate the quality of life of patients underwent laparoscopic and open colorectal resections in the early postoperative period. Methods: this is a prospective study which evaluated 42 patients underwent laparoscopic and open colorectal resection between May to November 2008 followed up until 60th postoperative day. Questionnaires of quality of life were applied in 3th, 7th and 30th postoperative days. Statistical analysis consisted of descriptive analysis of global healthy status scores, functional scores and symptoms of EORTC/QLQ 30. Shapiro-Wilk, Mann-Whitney e t de Student statistical tests were used to check the data, with level of significance in 0.05 . Results: Most of patients were females $(57.1 \%)$ with mean age of 61.5 years. It was observed significant difference of "global health status" score on the 30th postoperative between groups, with values of 75.0 and 58.3 for patients underwent laparoscopic and open procedures respectively $(p=0.005)$. There were no differences in terms of physical function and others as, accomplishment, emotional, cognitive and social functions. In relation to symptoms (fatigue, nausea, pain, dyspnea, insomnia, loss of appetite and constipation) and financial difficulties, there were also no differences between groups. Conclusion: Our results have demonstrated that patients underwent laparoscopic colorectal resections have better quality of life at the end of first postoperative month when compared to patients underwent to open colorectal resections.
}

Key words: Quality of life; colorectal cancer; laparoscopic surgery. 


\section{REFERÊNCIAS}

1. Abraham NS, Young JM, Solomon MJ. Meta-analysis of shortterm outcomes after laparoscopic resection for colorectal cancer. Br J Surg 2004; 91: 1111-24.

2. King PM, Blazeby JM, Ewings $\mathrm{P}$ et al. Randomized clinical trial comparing laparoscopic and open surgery for colorectal cancer within an enhanced recovery programme. Br J Surg 2006; 93: 300-8.

3. Janson M, Bjorholt I, Carlsson P et al. Randomized clinical trial of the costs of open and laparoscopic surgery for colonic cancer. Br J Surg 2004; 91: 409-17.

4. Schwenk W, Kehlet H. Meta-analysis of short-term outcomes after laparoscopic resection for colorectal cancer(Br J Surg 2004; 91: 1111-1124). Br J Surg 2004; 91: 1653-4.

5. Dowson HM, Cowie AS, Ballard K, et al. Systematic review of quality of life following laparoscopic and open colorectal surgery. Colorectal Disease 2008; 10: 757 -768.

6. Lourenco T, Murray A, Grant A, et al. Laparoscopic surgery for colorectal cancer: safe and effective? -A systematic review. Surg Endosc 2008; 22:1146-1160.

7. Fayers PM, ANBKGMCDBAobotEQoLSG. The EORTC QLQ-C30 Scoring Manual, 3rd edn. European Organisation for Research and Treatment of Cancer, Brussels 2001.
8. Schwenk W, Haase O, Neudecker J, Mu“ller JM. Short term benefits for laparoscopic colorectal resection. Cochrane Database Syst Rev 2005; Issue 3. Art. No.: CD003145. doi: 10.1002/14651858.CD003145.pub2.

9. Guillou PJ, Quirke P, Thorpe H et al. Short-term endpoints of conventional versus laparoscopic-assisted surgery in patients with colorectal cancer (MRC CLASICC trial): multicentre, randomised controlled trial. Lancet 2005; 365: 1718-26.

10. Weeks JC, Nelson H, Gelber S, et al. Clinical Outcomes of Surgical Therapy (COST) Study Group. Short-term qualityof-life outcomes following laparoscopic assisted colectomy vs open colectomy for colon cancer: a randomized trial. JAMA 2002; 16;287(3):321-328.

\section{Endereço para correspondência:}

\section{TEON AUGUSTO NORONHA DE OLIVEIRA}

Clínica Coloproctológica-HFR

Avenida do Contorno, 9.530

Belo Horizonte/MG

CEP: 30110-934

E-mail: teonaugusto@bol.com.br 